To be publisixed in Synthetic Metals

- Proceeding of International Conference on Synthetic Metals

Gastein, Ausitia July 15-21,2000

ANL/CHM/CP-101482A ,

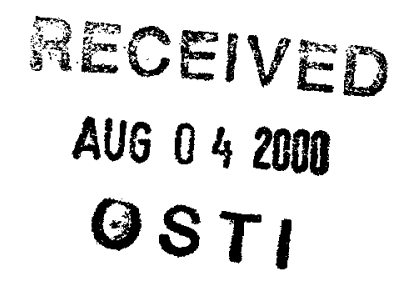

\title{
Two-dimensional magnetic quantum oscillations observed in an organic metal
}

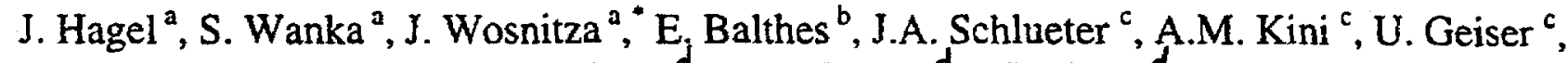 \\ J. Mohtasham ${ }^{d}$, R.W. Winter ${ }^{d}$, G.L. Gard ${ }^{d}$ \\ "Physikalisthes Instiut, Universität Karlsruhe. Engessersir. 7, D-76128 Karisruht, Germany \\ 'Grenoble High Maguetic Field Laboratory, MPI and C.N.R.S., 38042 Grenoble, France \\ 'Chemistry and Materials Science Divisions, Argonne National Laboraton, Argonne, Mlinois 604.39, USA \\ dDepartment of Chemistry, Porlland State University, Purtland, Oregon 97207. USA
}

Abstract

The de Haas-van Alphen (dHvA) signal of the organic superconducror $\beta$ "-(BEDT-TTF) ${ }_{2} \mathrm{SF}_{5} \mathrm{CH}_{2} \mathrm{CF}_{2} \mathrm{SO}_{3}$ shows an inverse-sawtooth wave form which proves the existence of an ideal two-dimensional (2D) Fermi surface. The dHvA wave shape can almost perfectly be described by a $2 D$ theory assuming a constant chemical potential. This either implies the existence of the predicted quasi-one-dimensional band with an exceptionally large density of states or the chemical potential may be pinned due to localized states near the Fermi energy.

Keywords: Organic superconductors. Magnetic measurements

Organic metals of the type (BEDT-TTF) $2 X$, where BEDT-TTF is bisechylenedithio-tetrathiafulvalene and $X$ represents a monovalent anion, are characterized by their pronounced two-dimensional (2D) electronic structure with, often, negligible dispersion perpendicular to the highly conducting planes [1]. Nevertheless, the de Haas-van Alphen (dHvA) oscillations in most of these high-quality metals exhibit only minor deviations from the conventional 3D Lifshitz-Kosevich (LK) theory [2]. This is in contrast to magnetoresistance oscillations where in a number of organic metals strong apparent deviations of the Shubnikovde Haas (SdH) signal from LK behavior have been reported (see references in [3]). In transport data, however, complicated scattering mechanisms are involved and a non-trivial tensor inversion is needed to extract the true SdH signal. Here, we report on true thermodynamic dHvA data which provide experimental evidence for the existence of a perfect 2D Fermi liquid in a bulk 3D organic metal.

The high-quality $\beta^{\prime \prime}$-(BEDT-TTF) $\mathrm{SF}_{3} \mathrm{CH}_{2} \mathrm{CF}_{2} \mathrm{SO}_{3}$ single crystals were grown by electrocrystallization [4]. The magnetization $M_{\perp}$ perpendicular to the applied magnetic field $B$ was measured by means of a cantilever torque magnetometer which was mounted on a rotatable sample holder in a ${ }^{3}$ He cryostat at the Grenoble High Magnetic Field Labora-

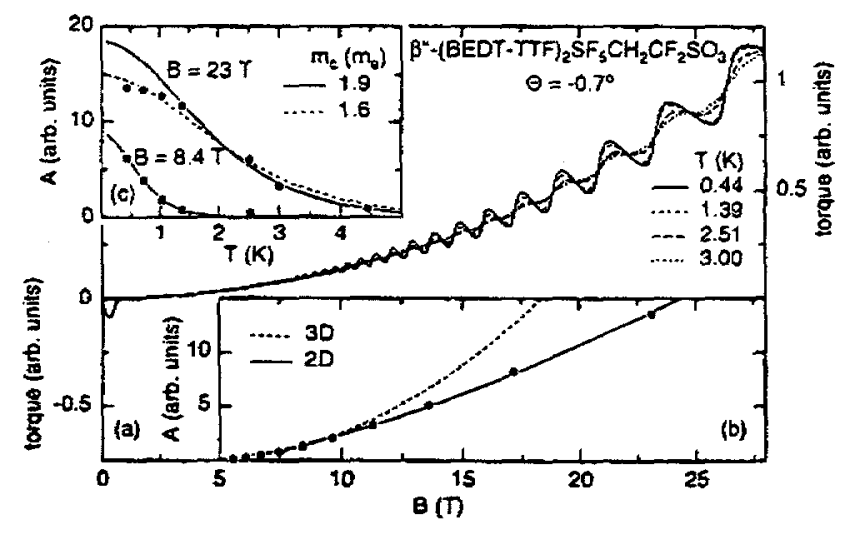

Fig. 1. (a) Fieid dependence of the torque signal of one high-guality sample for differeat temperatures. (b) Field dependence of the dHvA amplitude $A$ at $T=0.44 \mathrm{~K}$ with the field dependence expected from $3 \mathrm{D}$ LX theory (dashed line) and from the 2D theory (solid line). (c) Temperature dependence of $A$ for different average fields. The dashed line is a fit with the LK theory over all dota points at $B=23 \mathrm{~T}$, the solid lines are fits at higher temperatures or lower field.

tory. The torque is given by $\tau=M \times B$ which is proportional to $B^{2}$ for an anisotropic field-independent susceptibility $\chi$ of the sample. In general, $\chi$ is a tensor and the absolute value of the torque has the form $\tau \propto \Delta \chi B^{2} \sin 2 \theta$. 


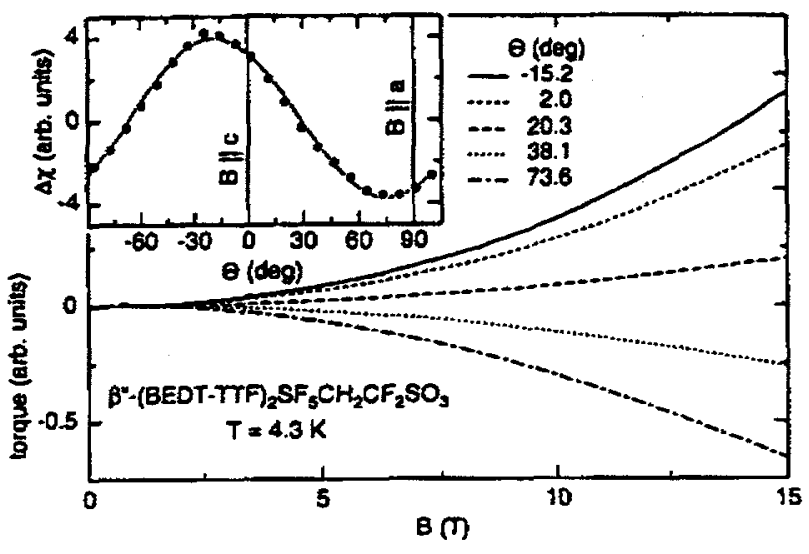

Fig. 2. Field dependence of the torque signal at $T=4.3 \mathrm{~K}$ for different angles. The angutar dependence of the susceptibility difference (inset) perfectly follows the expected $\sin 2 \theta$ dependence (solid line).

where $\Delta \chi$ is the susceptibility difference between two principal axes of the $\chi$ tensor and $\vartheta$ is the angle between $B$ and one of the principal tensor axes. For a 2D Fermi surface the torque dHvA signal is proportional to $d F / d \theta$, with $F=$ $F_{0} / \cos \Theta$ and the angle $\Theta$ between $B$ and the normal to the conducting planes.

Fig. 1(a) shows the torque signal at $\Theta=-0.7^{\circ}$ where the dHVA amplitude is very small and any nonlinearities of the torque signal can be excluded. The dHvA oscillations are sitting on a torque background which follows a $B^{2}$ behavior up to the highest field. This background signal is up to $T=$ $75 \mathrm{~K}$ almost temperature independent and shows a $\sin 2 \vartheta$ dependence as expected for a static field-independent susceptibility (Fig. 2). One principal axis of the $\chi$ tensor lies at $\theta=30^{\circ}$. The occurrence of this anisotropic susceptibility is rather unusual for the organic metals and the origin of the signal in the present material is unclear. It might be a remnant of the local magnetism of the molecular bands.

At low fields and high temperatures the dHvA data (Fig. 1) are in line with previous results [5] and the conventional LK behavior. However, at higher fields clear deviations from this 3D theory are detected. The dHvA amplitude $A$ increases much less at high fields than predicted for a 3D metal [dashed line in Fig. 1(b)]. Further on, this leads to an apparent field-dependent effective mass $m_{c}$ [Fig. 1(c)], i.e., $m_{\mathrm{c}}=1.9 \mathrm{~m}_{\mathrm{c}}$ at low $B$ and high $T$ in good agreement with earlier low-field data [5], but smaller $m_{\mathrm{c}}$ values for lower $T$ and higher $B$. This behavior is even more apparent for the higher harmonics which yield e.g. $m_{c}=1.1 m_{c}$ for the second harmonic when analyzed with the LK formula at $23 \mathrm{~T}$.

A very remarkable feature of the data is the inversesawtooth wave form of the dHvA signal (Fig. 3, where the quadratic background is subtracted). For a 2D metal with fixed number of charge carriers (canonical ensemble) an oscillating chemical potential and a "normal" sawtooth wave form is expected [6,7]. However, for a 2D metal with a charge-carrier reservoir, i.e., with fixed chemical potential in the grand-canonical ensemble, exactly the wave shape as observed is predicted [7,8]. In the latter case, an analytic formula for the oscillating magnetization is [8]

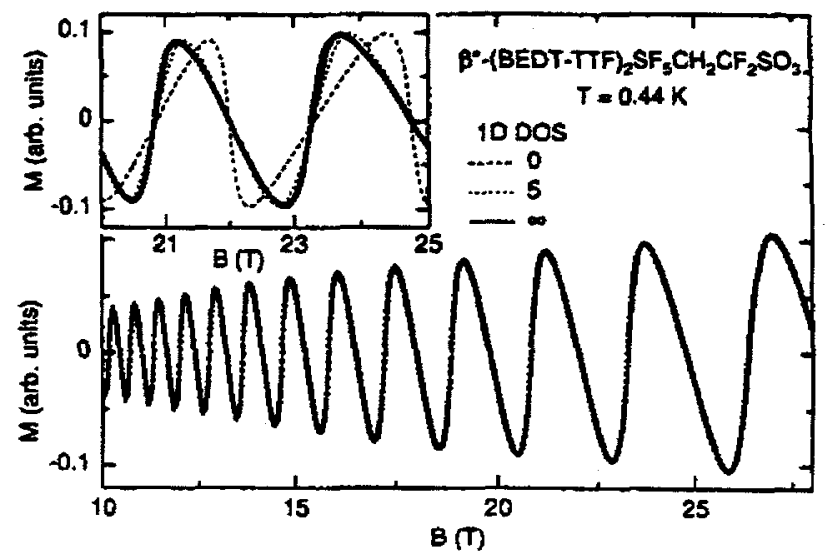

Fig. 3. The measured dHvA data (open symbols) follow the behavior for a 2D metal with fixed chemical potential [Eq. (1), solid line]. For a 2D metal with fixed number of charge-cariers sawtooth-like oscillations are expected (dished line in the inset). The dHvA wave shape for a charge. carrier seservoir with a density of states five times larger than for the $2 D$ band describes the data not yet perfectly (short-dashed line).

$$
M \propto \sum_{r=1}^{\infty} r^{-1} R_{\mathrm{D}} R_{\mathrm{T}} R_{S} \sin [2 \pi r(F / B-0.5)]
$$

with the Dingle $\left(R_{\mathrm{D}}\right)$, temperature $\left(R_{\mathrm{T}}\right)$, and spin-splitting factors $\left(R_{\mathrm{S}}\right)$ being identical to those in the 3D LK formula [8]. Almost perfect agreement between the dHvA data and the theory (1) with the parameters extracted at low fields and high temperatures is obtained (solid line in Fig. 3).

The observed inverse-sawtooth wave shape of the dHvA oscillations implies the existence of a fixed chemical potential. This either may occur through the transfer of electrons from magnetic-field independent states, like the predicted quasi-1D bands, or due to the existence of localized electron states near the Fermi surface [9]. Indeed, the latter scenario seems to be somewhat more likely, since otherwise a rather high density of states (DOS) originating from the ID bands has to be assumed in order to account for the observed wave shape. Using the numerical model of [7] with a ID DOS five times larger than for the 2D states we still cannot describe the dHvA data satisfactory (shortdashed line in the inset of Fig. 3).

\section{References}

[1] T. Ishiguro, K. Yamaji, and G. Saito, Organic Superconductors (Springer, Berlin, 1998) 2nd ed.

[2] J. Wosnitza. Fermi Surfaces of Low-Dimensional Organic Metals and Superconductors (Springer, Berlin, 1996).

[3] J. Wosnilza et al., Phys. Rev. B 61 (2000) 7383.

[4] U. Geiser et at., J. An Chem. Soc. 118 (1996) 9996; J.A. Schlueter at al., Synth. Met. 85 (1997) 1453.

[S] D. Beckmann et al.. Eur. Ptyys. J. B 6 (1998) 329; J. Wosnitza el al., Physica B 246-247 (1998) 104.

[6] 1.D. Vagner et al. Phys. Rev. Lete. 51 (1983) 1700.

[7) N. Harrison et al. Phys. Rev. B 54 (1996) 9977.

[8] D. Shoenberg, Magnetic Oscillations in Metsls (Cambridge University Press, Cambridge, 1984).

[9] M.A. liskovsky et al., Phys. Rev. B 61 (2000) 14610. 


\section{DISCLAIMER}

This report was prepared as an account of work sponsored by an agency of the United States Government. Neither the United States Government nor any agency thereof, nor any of their employees, make any warranty, express or implied, or assumes any legal liability or responsibility for the accuracy, completeness, or usefulness of any information, apparatus, product, or process disclosed, or represents that its use would not infringe privately owned rights. Reference herein to any specific commercial product, process, or service by trade name, trademark, manufacturer, or otherwise does not necessarily constitute or imply its endorsement, recommendation, or favoring by the United States Government or any agency thereof. The views and opinions of authors expressed herein do not necessarily state or reflect those of the United States Government or any agency thereof. 


\section{DISCLAIMER}

Portions of this document may be illegible in electronic image products. Images are produced from the best available original document. 